\title{
Topology Changing Process of Coalescing Black Holes on Eguchi-Hanson Space
}

\author{
${ }^{1}$ Masashi Kimura*, ${ }^{1}$ Hideki Ishihara ${ }^{\dagger},{ }^{2}$ Shinya Tomizawa ${ }^{\ddagger}$ \\ and ${ }^{3}$ Chul-Moon $\mathrm{Yoo}^{\S}$ \\ ${ }^{1}$ Department of Mathematics and Physics, \\ Graduate School of Science, Osaka City University, \\ 3-3-138 Sugimoto, Sumiyoshi, Osaka 558-8585, Japan \\ ${ }^{2}$ Cosmophysics Group, Institute of Particle and Nuclear Studies, \\ KEK, Tsukuba, Ibaraki, 305-0801, Japan \\ ${ }^{3}$ Asia Pacific Center for Theoretical Physics, \\ Pohang University of Science and Technology, Pohang 790-784, Korea
}

(Dated: November 5, 2018)

\begin{abstract}
We numerically study the event horizons of two kinds of five-dimensional coalescing black hole solutions with different asymptotic structures: the five-dimensional Kastor-Traschen solution (5DKT) and the coalescing black hole solution on Eguchi-Hanson space (CBEH). Topologies of the spatial infinity are $S^{3}$ and $L(2 ; 1)=S^{3} / \mathbb{Z}_{2}$, respectively. We show that the crease sets of event horizons are topologically $\mathrm{R}^{1}$ in $5 \mathrm{DKT}$ and $\mathrm{R}^{1} \times \mathrm{S}^{1}$ in $\mathrm{CBEH}$, respectively. If we choose the time slices which respect space-time symmetry, the first contact points of the coalescing process is a point in the $5 \mathrm{DKT}$ case but a $\mathrm{S}^{1}$ in the $\mathrm{CBEH}$ case. We also find that in $\mathrm{CBEH}$, time slices can be chosen so that a black ring with $\mathrm{S}^{1} \times \mathrm{S}^{2}$ topology can be also formed during a certain intermediate period unlike the 5DKT.
\end{abstract}

PACS numbers: 04.50.+h, 04.70.Bw

\footnotetext{
* E-mail:mkimura@sci.osaka-cu.ac.jp

$\dagger$ E-mail:ishihara@sci.osaka-cu.ac.jp

$\ddagger$ E-mail:tomizawa@post.kek.jp

$\S$ E-mail:c_m_yoo@apctp.org
} 


\section{INTRODUCTION}

One of the most interesting predictions of string theory, which is a strong candidate of the unified theory, is that our world is a higher dimensional space-time. Since we feel we live in the four-dimensional space-time in low energy physical phenomena, extra-dimensions should be effectively compactified. It is natural to consider that the space-time is locally a direct product of our four-dimensional space-time and extra-dimensions. However, there are many possibilities how four-dimensional space-time and extra-dimensions are connected globally. The structure of the whole higher-dimensional space-time is not known.

Can we get any information of the global structure of the whole higher-dimensional spacetime from any experiment localized in a finite region? To approach this question, at the first step, we consider a system of black holes with a non-trivial asymptotic structure as a toy model and we compare the coalescing process with a trivial asymptotic structure case.

In the series of works [1, 2, 3], it is presented that differences of black hole coalescence processes caused by asymptotic structure. In the case of the five-dimensional space-time in which the boundary of the spatial infinity is not $S^{3}$ but lens space, it is possible that two black holes with the topology of $\mathrm{S}^{3}$ coalesce and change into a single black hole with the topology of the lens space, i.e., $\mathrm{S}^{3}+\mathrm{S}^{3} \rightarrow L(2 ; 1)=\mathrm{S}^{3} / \mathbb{Z}_{2}$. Analyzing the horizons at early time and late time, we found that the areas of the horizon after coalescence in such cases is larger than the ordinary cases in five dimension, i.e., $S^{3}+S^{3} \rightarrow S^{3}$. However, the shape of the event horizon is not clear in an intermediate period. In this paper, we discuss details of event horizon structures in coalescing black holes with non-trivial asymptotic structures. ${ }^{1}$

The paper is organized as follows: in Section II we briefly review the coalescing black holes with trivial and non-trivial asymptotic structures in five dimensions. Section III we investigate the structures of the event horizon of coalescing black holes numerically and discuss coalescing process in typical time slices. Section IV is devoted to the discussion of the structure of the crease set of the event horizon. Finally, in Section V we discuss the obtained results.

\footnotetext{
1 Recently, the structure of the event horizons of lens space was also discussed in [4].
} 


\section{COALESCING BLACK HOLES IN FIVE DIMENSIONS}

Black hole coalescence is one of the most interesting issues in a study of gravity. To treat the coalescencing process, we need heavy numerical work in general. However, if the mass and the electric charge of each black hole are equal, we can construct exact solutions which describe the coalescencing processes driven by a positive cosmological constant [1, $\mathbf{3}, \underline{5}, \underline{6}$, 7, 8, 9, 10].2.

One of such solutions in five-dimensional Einstein-Maxwell theory with a positive cosmological constant is the Kastor-Traschen solution(5DKT) [5, 6]. The metric and gauge 1-form for 5DKT are given by

$$
\begin{aligned}
d s^{2} & =-H^{-2} d t^{2}+H e^{-\lambda t}\left[d x^{2}+d y^{2}+d z^{2}+d w^{2}\right] \\
A & = \pm \frac{\sqrt{3}}{2} H^{-1} d t
\end{aligned}
$$

with

$$
H=1+\frac{1}{e^{-\lambda t}}\left(\frac{m_{1}}{x^{2}+y^{2}+z^{2}+(w-a)^{2}}+\frac{m_{2}}{x^{2}+y^{2}+z^{2}+(w+a)^{2}}\right),
$$

respectively, where $\lambda=2 \sqrt{\Lambda / 3}$ and $\Lambda$ is a positive cosmological constant. This solution describes the physical process such that two black holes with the topology of $S^{3}$ coalesce into a single black hole with the topology of $S^{3}$.

Recently, coalescing black hole solution on Eguchi-Hanson space(CBEH) [1] has been found as another exact solution in the same theory. The metric and gauge 1-form for this solution are given by

$$
\begin{aligned}
d s^{2} & =-H^{-2} d t^{2}+H e^{-\lambda t}\left[V^{-1}\left(d x^{2}+d y^{2}+d z^{2}\right)+V((a / 8) d \psi+\omega)^{2}\right], \\
A & = \pm \frac{\sqrt{3}}{2} H^{-1} d t
\end{aligned}
$$

2 If we set the cosmological constant to zero, the solution [5, 6] reduces to the Majumdar-Papapetrou solution [11, 12, 13] which describes static multi-black holes. The higher-dimensional generalizations of the multi-black holes are discussed in [14, 15, 16, 17], and recently the smoothness of horizons of higher-dimensional multi-black holes are also investigated in [18, 19, 20, 21]. 
with

$$
\begin{aligned}
H & =1+\frac{1}{e^{-\lambda t}}\left(\frac{M_{1}}{\sqrt{x^{2}+y^{2}+(z-a)^{2}}}+\frac{M_{2}}{\sqrt{x^{2}+y^{2}+(z+a)^{2}}}\right), \\
V^{-1} & =\frac{a / 8}{\sqrt{x^{2}+y^{2}+(z-a)^{2}}+\frac{a / 8}{\sqrt{x^{2}+y^{2}+(z+a)^{2}}},} \\
\omega & =\frac{a}{8}\left[\frac{z-a}{\sqrt{x^{2}+y^{2}+(z-a)^{2}}}+\frac{z+a}{\sqrt{x^{2}+y^{2}+(z+a)^{2}}}\right] \frac{x d y-y d x}{x^{2}+y^{2}},
\end{aligned}
$$

where we note that the metric inside the square bracket in (44) is the metric of the EguchiHanson space [22, 23] which has a non-trivial asymptotic structure called the lens space $L(2 ; 1)=\mathrm{S}^{3} / \mathbb{Z}_{2}$.

This solution describes the physical process such that two black holes with the topology of $\mathrm{S}^{3}$ coalesce into a single black hole with the topology of the lens space $L(2 ; 1)$ due to the non-trivial asymptotic structure [1]. To confirm this, let us see the behavior of the metric at early time $t \rightarrow-\infty$ and at late time $t \rightarrow \infty$, following the discussion in [1]. At early time $t \rightarrow-\infty$ and $R_{i} \rightarrow 0$, the metric behaves as

$$
\begin{aligned}
d s^{2} \simeq & -\left(1+\frac{m_{i}}{e^{-\lambda t} r_{i}^{2}}\right)^{-2} d t^{2} \\
& +\left(1+\frac{m_{i}}{e^{-\lambda t} r_{i}^{2}}\right) e^{-\lambda t}\left[d r_{i}^{2}+\frac{r_{i}^{2}}{4}\left(d \theta^{2}+\sin ^{2} \theta d \phi^{2}+(d \psi+\cos \theta d \phi)^{2}\right)\right],
\end{aligned}
$$

where we have introduced a new radial coordinate, $r_{i}^{2}:=R_{i} a / 2$ and a new mass parameter, $m_{i}:=M_{i} a / 2$. This metric is the same form as that of five-dimensional Reissner-Nordströmde Sitter solution with mass parameter $m_{i}$ written in the cosmological coordinate. Therefore, we can see that there are two black holes with the topology of $\mathrm{S}^{3}$ at early time. On the other hand, at late time $t \rightarrow \infty$ and $R \rightarrow \infty$, the metric behaves as

$$
\begin{aligned}
d s^{2} & \simeq-\left(1+\frac{2\left(m_{1}+m_{2}\right)}{r^{2}}\right)^{-2} d t^{2} \\
& +\left(1+\frac{2\left(m_{1}+m_{2}\right)}{e^{-\lambda t} r^{2}}\right) e^{-\lambda t}\left[d r^{2}+\frac{r^{2}}{4}\left(d \theta^{2}+\sin ^{2} \theta d \phi^{2}+\left(\frac{d \psi}{2}+\cos \theta d \phi\right)^{2}\right)\right]
\end{aligned}
$$

where we introduce the new radial coordinate $r^{2}:=a R$. This resembles the metric of the five-dimensional Reissner-Nordström-de Sitter solution with mass equal to $2\left(m_{1}+m_{2}\right)$ but the topology of horizon is the lens space $L(2 ; 1)$. Therefore, we can see the solution (4) describes the process such that two black holes with $\mathrm{S}^{3}$ at early time coalesce into a single black hole with the lens space $L(2 ; 1)$ at late time. 


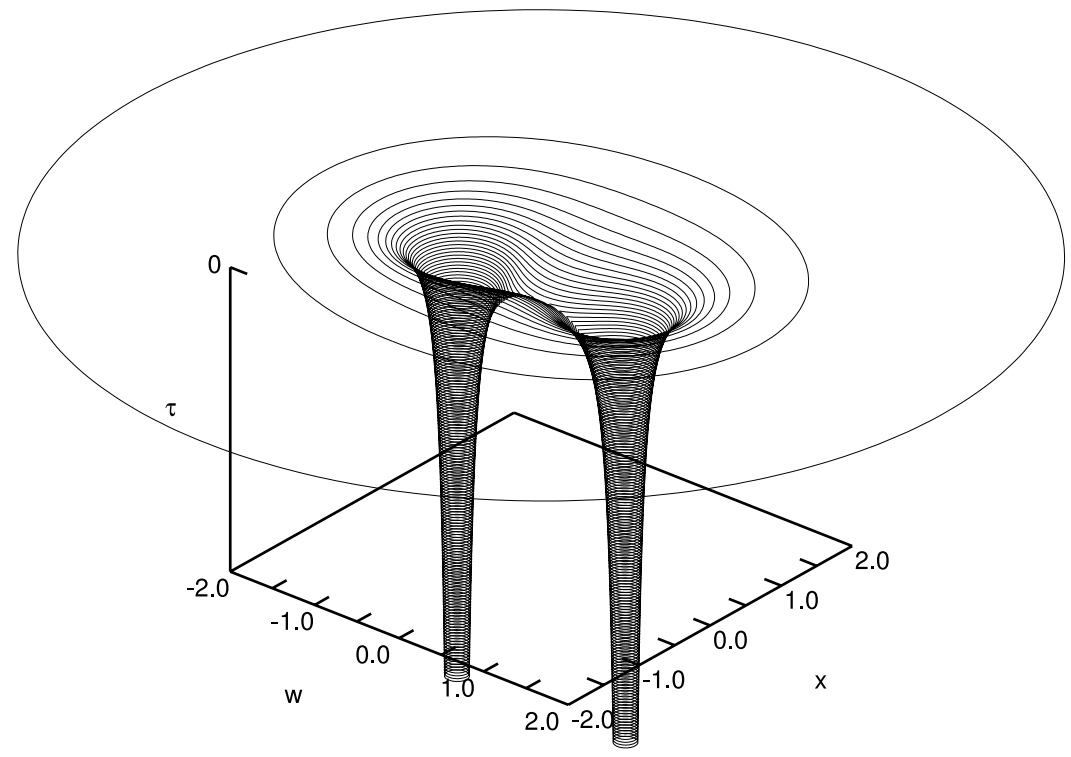

FIG. 1: Event horizon of five dimensional Kastor-Traschen solution. $\left(m_{1}=m_{2}=1, a=1, \lambda=\right.$ $1 /(2 \sqrt{2}))$

However, since here, we only investigate the asymptotic behaviors of the metric at early time and at late time, it is not clarified how two black holes with $\mathrm{S}^{3}$ coalesce into a single black hole with the lens space $L(2 ; 1)$. So, in the following sections, we numerically investigate the location and the shape of the event horizon for the solution (44)-(8) and make clear the process of the coalescence.

\section{EVENT HORIZONS}

An event horizon is defined as the boundary of the causal past of the future null infinity. Due to the absence of Killing horizons in a dynamical spacetime, it is difficult to determine the location of an event horizon analytically. We can investigate the location of the event horizon by integrating numerically null geodesic equations backward from sufficiently future to the past [9].

Since both metrics Eqs.(11) and (4) asymptote to the metric of Reissner-Nordström-de Sitter solution in the limit of $t \rightarrow \infty$, fortunately we can know where the event horizons locate at late time. So if we solve null geodesic equations numerically from each point of the spatial cross section of the event horizons at late time backward the past where we set the 

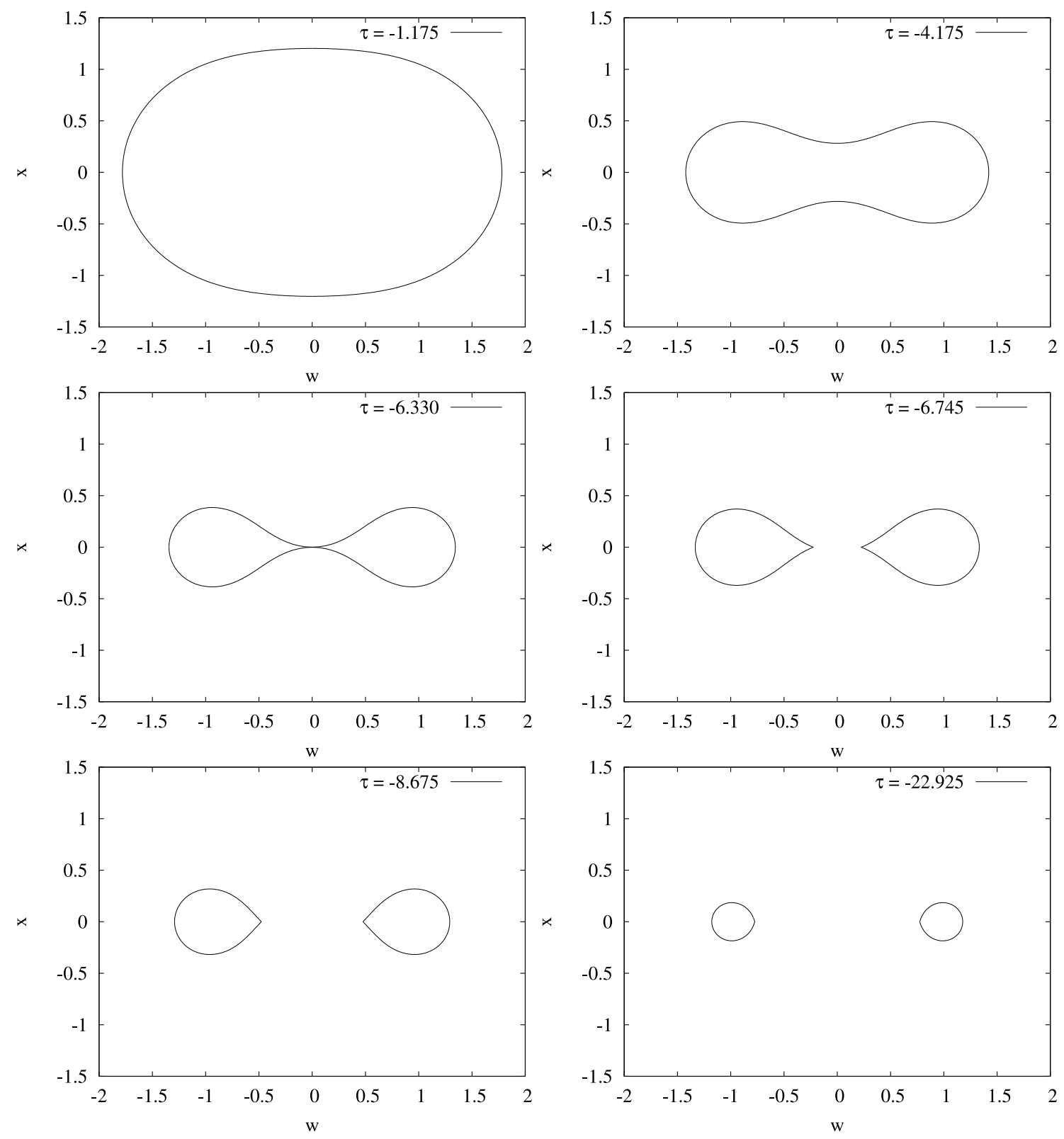

FIG. 2: Coordinate value of event horizon of each time slices in five dimensional Kastor-Traschen solution.

initial null direction to be tangent to the event horizon at late time, we can get null geodesic generators of the event horizons, namely we can find the locations of the event horizons. 


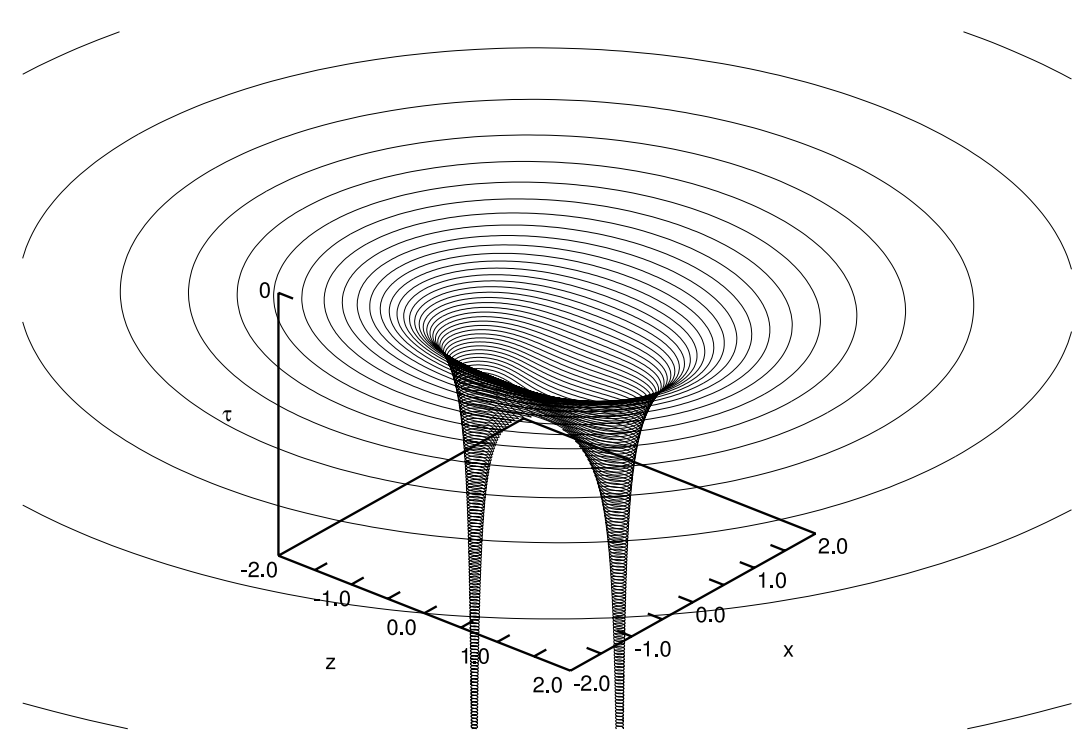

FIG. 3: Event horizon of coalescing black holes on Eguchi-Hanson space. $\left(M_{1}=M_{2}=2, a=\right.$ $1, \lambda=1 /(2 \sqrt{2}))$

\section{A. 5DKT case}

In 5DKT case, we obtain null geodesic generators of the event horizon for the metric (11). We can set $y=0, z=0$ without loss of generality because of the $S O(3)$ symmetry in $(x, y, z)$ space. Then, we solve null geodesics in the effective three-dimensional metric given by

$$
\begin{aligned}
d s^{2} & =-H^{-2} d t^{2}+H e^{-\lambda t}\left[d x^{2}+d w^{2}\right] \\
H & =1+\frac{1}{e^{-\lambda t}}\left(\frac{m_{1}}{x^{2}+(w-a)^{2}}+\frac{m_{2}}{x^{2}+(w+a)^{2}}\right) .
\end{aligned}
$$

After some numerical calculations, we get the data of coordinate values of location of event horizon, and we plot the coordinate values $\tau:=e^{-\lambda t}, x, w$ in Fig! In this graph, each contour line means cross section of horizon with a $\tau=$ const. surface. In Fig, 2 , we plot the the coordinate values $x, w$ of event horizon at some typical time slices. From Fig 2 we can see that two black holes collide at $\tau=-6.330$ and the first contact point is given by $x=0, w=0$. Since $w$-axis is fixed points $S O(3)$ symmetry in $(x, y, z)$ space, the topology of this first contact point in Fig.2 is a point in the whole five-dimensional space-time. 

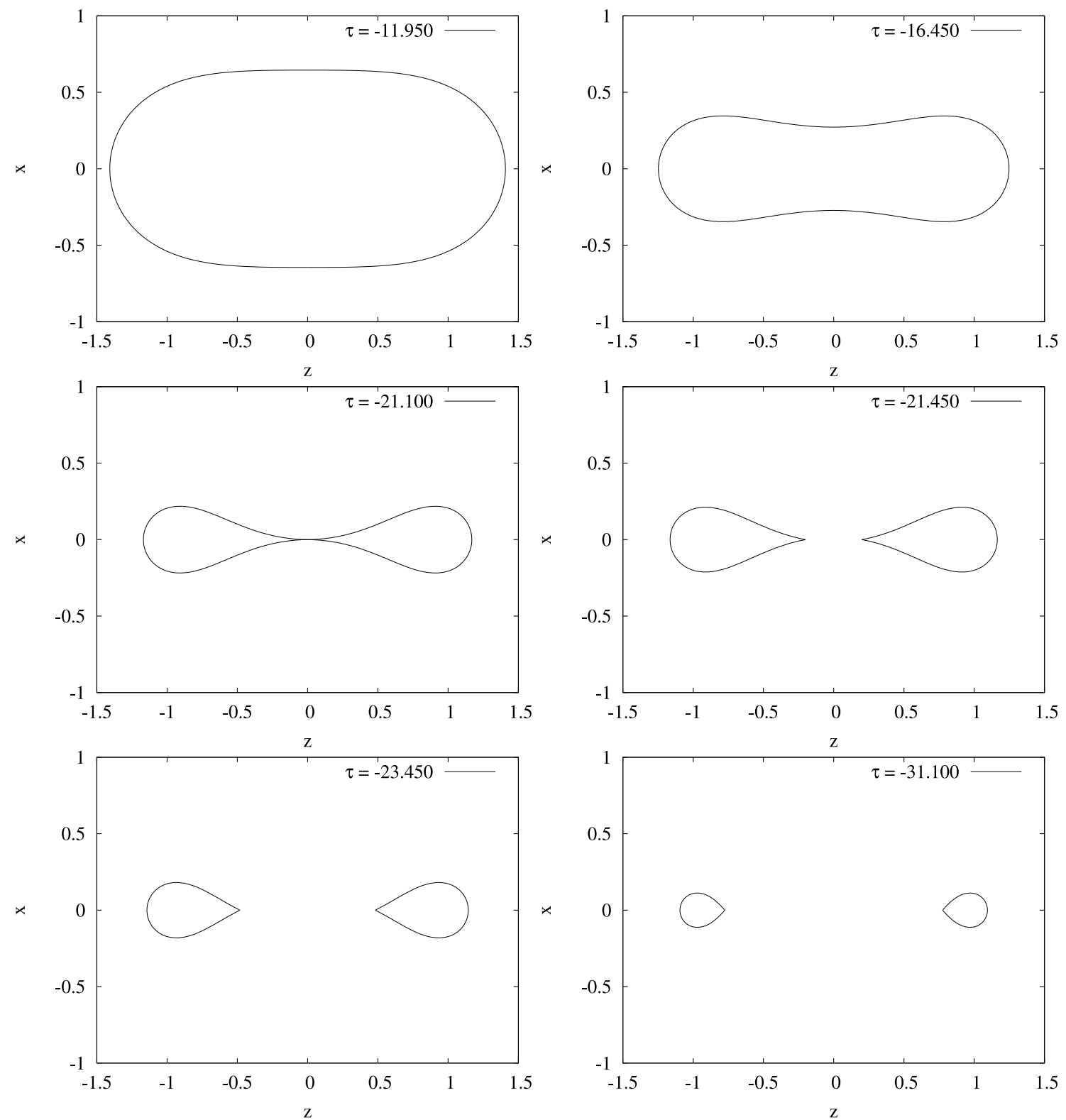

FIG. 4: Coordinate value of event horizon of each time slices in coalescing black holes on EguchiHanson space.

\section{B. CBEH case}

In $\mathrm{CBEH}$ case, we obtain null geodesic generators of the event horizon for the metric (44). We can set $y=0$ without loss of generality because of the $S O(2)$ symmetry in $(x, y)$ space. Furthermore, since $\partial_{\psi}$ is a Killing vector, we can set $\psi=0$. Then, we solve null geodesics 
in the effective three-dimensional metric given by

$$
\begin{aligned}
d s^{2} & =-H^{-2} d t^{2}+H e^{-\lambda t} V^{-1}\left(d x^{2}+d z^{2}\right) \\
H & =1+\frac{1}{e^{-\lambda t}}\left(\frac{M_{1}}{\sqrt{x^{2}+(z-a)^{2}}}+\frac{M_{2}}{\sqrt{x^{2}+(z+a)^{2}}}\right), \\
V^{-1} & =\frac{a / 8}{\sqrt{x^{2}+(z-a)^{2}}}+\frac{a / 8}{\sqrt{x^{2}+(z+a)^{2}}} .
\end{aligned}
$$

Similar to the above discussion, we plot the coordinate values of event horizon $\tau:=e^{-\lambda t}, x, z$ in Fig 3, In Fig, 4, we plot the the coordinate values $x, z$ of event horizon at some typical time slices. From Fig, we can see that two black holes collide at $\tau=-21.100$ and the first contact point is given by $x=0, z=0$. In this case, $z$-axis is fixed points of $S O(2)$ symmetry in $(x, y)$ space, but $\tau=-21.100, x=0, z=0$ is not a fixed point of the $U(1)$ isometry generated by $\partial_{\psi}$. So the topology of this first contact point in Fig 4 is not a point but $\mathrm{S}^{1}$ in the whole five-dimensional space-time since the integral curve of $\partial_{\psi}$ is $\mathrm{S}^{1}$. This is the specific difference from 5DKT.

\section{CREASE SET AND TIME SLICE DEPENDENCE}

The intermediate time evolutions of the spatial cross section of black hole coalescence depends on the choice of time slices in general. As discussed in [4, 24, 25], how black holes coalesce is determined invariantly by the structure of the crease set of an event horizon and the spatial horizon topology far in the future, where the crease set is a set of past end points of null geodesic generators of an event horizon and the event horizon cannot be smooth there.

In $5 \mathrm{DKT}$, the crease set is given by the form of $\tau=\tau(w), x=y=z=0,-a<w<a$. Hence we immediately find that the topology of the crease set is $\mathrm{R}^{1}$. On the other hand, in $\mathrm{CBEH}$, the crease set is given by the form of $\tau=\tau(z), x=y=0,-a<z<a$, which indicates $\mathrm{R}^{1} \times \mathrm{S}^{1}$ in the space-time, where $\mathrm{S}^{1}$ is generated by $\partial_{\psi}$. Clearly, the dimension of crease sets in 5DTK and CBEH are different. For this reason, choosing the $\tau=$ constant slice before the coalescence occur and focusing on one black hole, the spatial sections of the event horizons can be schematically drawn as in Figs,5, 6, respectively.

To see the explicit difference between intermediate evolutions, we consider another time slice (i) shown in Fig:7 for the both 5DKT and CBEH cases. For simplicity, we assume the 


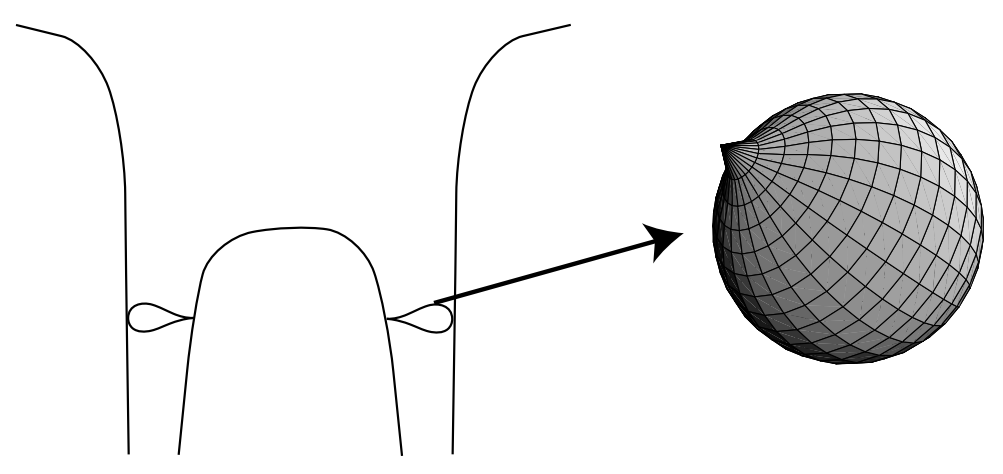

FIG. 5: Schematic figure of the event horizon of 5DKT focused on one black hole in some time slice before the coalescence occur. The topology of the spatial cross section of the crease set is a point.

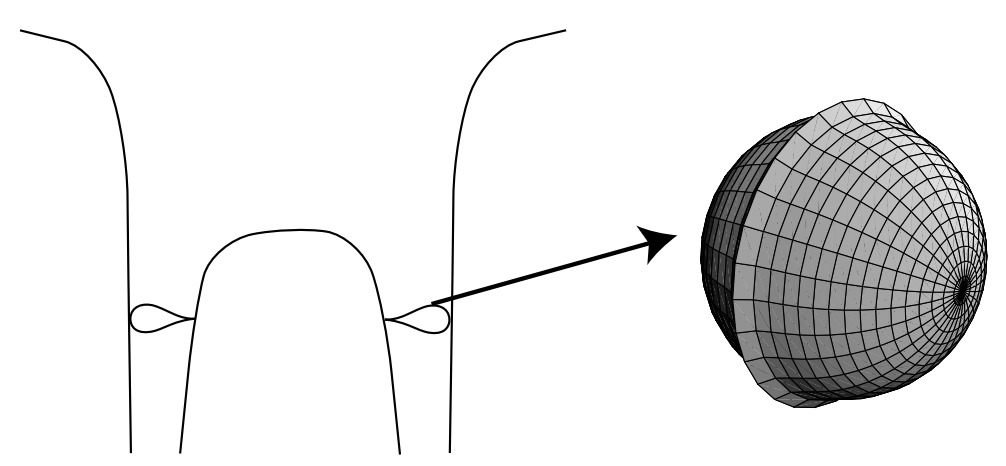

FIG. 6: Schematic figure of the event horizon of CBEH focused on one black hole in some time slice before the coalescence occur. The topology of the spatial cross section of the crease set is $\mathrm{S}^{1}$ which correspond to a great circle of $\mathrm{S}^{3}$.

spatial symmetry is the same as $\tau=$ const. surface. The central part of the intersection of the slice (i) and the event horizon makes a three-dimensional closed surface in the both cases.

In the case of $5 \mathrm{DKT}$, because the points $A$ and $B$ in Fig:7 are fixed points of the $\mathrm{SO}(3)$ rotation in $(x, y, z)$ space, the middle closed surface in Fig.7 is topologically $\mathrm{S}^{3}$, i.e., the middle region is a black hole with $\mathrm{S}^{3}$ horizon. For more general time slices, a number of black holes with $\mathrm{S}^{3}$ horizons can appear in the 5DKT case as the time slice (ii) shown in Fig.7.

In contrast, in the case of $\mathrm{CBEH}$, because the points $A$ and $B$ in Fig 7 are fixed points of 

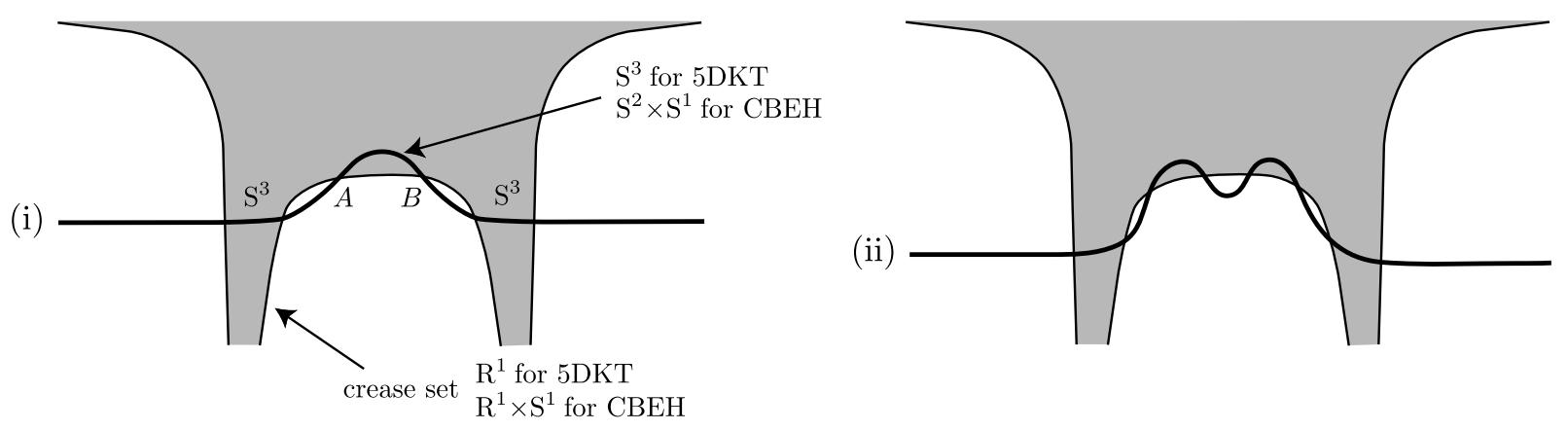

FIG. 7: Schematic figure of the side view of the event horizon of 5DKT and CBEH. Typical time slices (i) and (ii) which are different from the time slice $\tau$ are depicted. We assume time slices (i) and (ii) respect $S O(3)$ symmetry for $5 \mathrm{DKT}$ and $S O(2) \times U(1)$ symmetry for CBEH.

the $S O(2)$ rotation but not fixed points of the $U(1)$ generated by $\partial_{\psi}$, the intersection of the slice (i) and the event horizon is topologically $\mathrm{S}^{1} \times \mathrm{S}^{2}$. Then a black ring $\mathrm{S}^{1} \times \mathrm{S}^{2}$ is formed in the time slice (i) during coalescence of two black holes in the CBEH case. This is due to the differences of the structure of the crease set.

\section{SUMMARY AND DISCUSSION}

In this paper, we have studied how the structures of the event horizons of five-dimensional coalescing black holes differ in association with the asymptotic structure. We especially focus on the two solutions, i.e. Kastor-Traschen solution(5DKT) [5, 6] in which two black holes with $\mathrm{S}^{3}$ topology coalesce into a single black hole with $\mathrm{S}^{3}$ topology and coalescing black hole solution on Eguchi-Hanson space(CBEH)[1] in which two black holes with $\mathrm{S}^{3}$ topology coalesce into a single black hole with the lens space $L(2 ; 1)$ topology. It came out that, if we choose the time slices in which the symmetry of the base space is respected, the first contact points of the coalescing process compose $\mathrm{S}^{1}$ in the CBEH case unlike the 5DKT case in which the first contact point is a point in the five dimensional spacetime. This is the specific difference in topology changing process $\mathrm{S}^{3}$ into the lens space $L(2 ; 1)$ in the $\mathrm{CBEH}$ case.

The fact the first contact point is $\mathrm{S}^{1}$ in $\mathrm{CBEH}$ can be understood as follows. Let us consider the coalescence of two spheres centered at each nut of the Eguchi-Hanson space which is the base space of $\mathrm{CBEH}$ instead of considering the coalescence of black holes. If 
the two spheres are chosen to be symmetric the coalescence would occur at the midpoint between the two nuts. The topology of the midpoint between the two nuts is $\mathrm{S}^{1}$ which corresponds to equator of $\mathrm{S}^{2}$-bolt of the Eguchi-Hanson space in which two nuts are on the north and the south pole respectively.

We have also investigated the structure of crease sets of the event horizons. We have shown that the topologies of crease sets are $\mathrm{R}^{1}$ for $5 \mathrm{DKT}$ and $\mathrm{R}^{1} \times \mathrm{S}^{1}$ for CBEH. Since the dimensions of the crease sets are different from each other, it is expected that we can see the difference of intermediate evolutions explicitly if we adopt a different timeslice. In fact, we can find the time slice in which black ring $S^{1} \times S^{2}$ is formed in a certain period in the case of CBEH unlike the 5DKT.

\section{Acknowledgments}

We would like to thank Masaru Siino and Ken-ichi Nakao for useful discussions. This work is supported by the JSPS Grantin-Aid for Scientific Research No. 19540305. MK is supported by the JSPS Grant-in-Aid for Scientific Research No. 20-7858. ST is supported by the JSPS under Contract No. 20-10616.

[1] H. Ishihara, M. Kimura and S. Tomizawa, Class. Quant. Grav. 23, L89 (2006) arXiv:hep-th/0609165].

[2] C. M. Yoo, H. Ishihara, M. Kimura, K. Matsuno and S. Tomizawa, Class. Quant. Grav. 25, 095017 (2008) arXiv:0708.0708 [gr-qc]].

[3] K. Matsuno, H. Ishihara, M. Kimura and S. Tomizawa, Phys. Rev. D 76, 104037 (2007) arXiv:0707.1757 [hep-th]].

[4] D. Ida, arXiv:0904.3581 [gr-qc].

[5] D. Kastor and J. H. Traschen, Phys. Rev. D 47, 5370 (1993) arXiv:hep-th/9212035.

[6] L. A. J. London, Nucl. Phys. B 434, 709 (1995).

[7] D. R. Brill, G. T. Horowitz, D. Kastor and J. H. Traschen, Phys. Rev. D 49, 840 (1994) arXiv:gr-qc/9307014.

[8] K. I. Nakao, T. Shiromizu and S. A. Hayward, Phys. Rev. D 52, 796 (1995). 
[9] D. Ida, K. i. Nakao, M. Siino and S. A. Hayward, Phys. Rev. D 58, 121501 (1998).

[10] M. Kimura, arXiv:0904.4311 [gr-qc].

[11] S. D. Majumdar, Phys. Rev. 72, 390 (1947).

[12] A. Papaetrou, Proc. Roy. Irish Acad. (Sect. A) A 51 (1947) 191.

[13] J. B. Hartle and S. W. Hawking, Commun. Math. Phys. 26, 87 (1972).

[14] R. C. Myers, Phys. Rev. D 35, 455 (1987).

[15] J. P. Gauntlett, J. B. Gutowski, C. M. Hull, S. Pakis and H. S. Reall, Class. Quant. Grav. 20, 4587 (2003) arXiv:hep-th/0209114].

[16] H. Ishihara, M. Kimura, K. Matsuno and S. Tomizawa, Class. Quant. Grav. 23, 6919 (2006) arXiv:hep-th/0605030].

[17] H. Ishihara, M. Kimura, K. Matsuno and S. Tomizawa, Phys. Rev. D 74, 047501 (2006) arXiv:hep-th/0607035.

[18] D. L. Welch, Phys. Rev. D 52, 985 (1995) arXiv:hep-th/9502146.

[19] G. N. Candlish and H. S. Reall, Class. Quant. Grav. 24, 6025 (2007) [arXiv:0707.4420 [gr-qc]].

[20] G. N. Candlish, arXiv:0904.3885 [hep-th].

[21] M. Kimura, Phys. Rev. D 78, 047504 (2008) [arXiv:0805.1125 [gr-qc]].

[22] T. Eguchi and A. J. Hanson, Phys. Lett. B 74, 249 (1978).

[23] G. W. Gibbons and S. W. Hawking, Phys. Lett. B 78, 430 (1978).

[24] M. Siino, Phys. Rev. D 58, 104016 (1998) arXiv:gr-qc/9701003.

[25] M. Siino, Prog. Theor. Phys. 99, 1 (1998) arXiv:gr-qc/9803099. 